\title{
Ion Beam Preparation Procedures for Three-dimensional SEM Resolved Kikuchi (EBSD) and Kossel Microdiffraction Analysis of Deformed Metals
}

\author{
W. Hauffe*, G. Simons**, K. Kunze***, E. Langer*, R.J. Mitro**** \\ *Physics Department, Dresden University of Technology, D-01062 Dresden, Germany \\ ** Institute of mechanical Systems, ETH Zürich, 8092 Zürich, Switzerland \\ ${ }^{* * *}$ Geological Institute, ETH Zürich, 8092 Zürich, Switzerland \\ **** Gatan R\&D, 5933 Coronado Lane, Pleasanton, CA 94588, USA
}

The SEM provides information not only on the sample surface and near-surface regions concerning topography, composition, crystal orientation etc. With special preparation techniques the full $3 \mathrm{D}$ microstructure can be detected. The deformation-free revealing of the internal structure is not possible with mechanical cutting and grinding. Chemical and electrolytic methods allow only selected material-specific solutions. Ion beam preparation has essential advantages compared with conventional techniques. Especially for mechanically deformed metal samples with small dimensions special ion beam processing steps are required. The well established FIB technology is only useful for very small selected regions with micrometer dimensions. Here the problem will be solved to cut and to investigate samples with cross sections of typically $20 \mu \mathrm{m}$ x $400 \mu \mathrm{m}$ and $4 \mathrm{~mm}$ length after well defined deformation by microscopy and microdiffraction in the SEM over the full sample volume. The sample shape and size are shown in Fig. 1. After defined tensile tests these samples have been cut longitudinally (cut 1) and transverse (cut 2) by ion beam slope cutting and the macroarea was etched chemically (region 3). The ion beam cutting method [1] was carried out with the Gatan Precision Etching Coating System (PECS) acc. to Fig. 2. The broad ion beam is directed onto the sample mounted under a blind with a sharp edge. The ion gun allows to produce a beam of inert gas or reactive ions with energies up to $10 \mathrm{keV}$ and densities up to $40 \mu \mathrm{A} / \mathrm{mm}^{2}$. The processes can be observed by optical microscopy. A new stage allows sample positioning, tilting and rotation, blind mounting and adjustment with accurate sample transfer into the SEM for the final inspection. Fig. 3 shows a detail of the longitudinal ion beam cut area. In Fig. 4 the ion beam cut area of the full cross section (transversal cut) is shown. The cutting steps were carried out with $7 \mathrm{keV}$ Krypton ions. The texture analysis by EBSD is shown in Fig. 5 for the cuts corresponding to Fig. 1 by Orientation Imaging Microscopy (OIM) maps of a $20 \mu \mathrm{m}$ thin rolled $\mathrm{Cu}$ foil after a tensile test. More detailed discussion of the texture modified by deformation will be given in [2]. Also X-ray Kossel microdiffraction pattern have been detected of a tensile deformed Ni crystal [3]. The Kossel pattern in Fig. 6 shows an example of strong broadening and anisotropic intensity change of reflections due to the deformation process. EBSD provides information on crystallographic orientations, whereas X-ray Kossel microdiffraction pattern allow profound statements of the real microstructure. For both techniques the ion beam procedures are excellent tools to produce cut areas with high accuracy and to combine it very well with additional analysing methods.

\section{References}

[1] W. Hauffe, Production of Microstructures by Ion Beam Sputtering, Chapter 6 in Sputtering by Particle Bombardment III (Eds. R.Behrisch and K.Wittmaack), Springer Ser. Topics in Applied Physics, Vol. 64 (Springer-Verlag Heidelberg New York (1991) 305

[2] G. Simons, K. Kunze, W. Hauffe, J. Dual, Solid State Phenomena (accepted for publication) [3] E. Langer, S. Däbritz, W. Hauffe, Microsc. Microanalysis 9 (Suppl 3) (2003) 114 


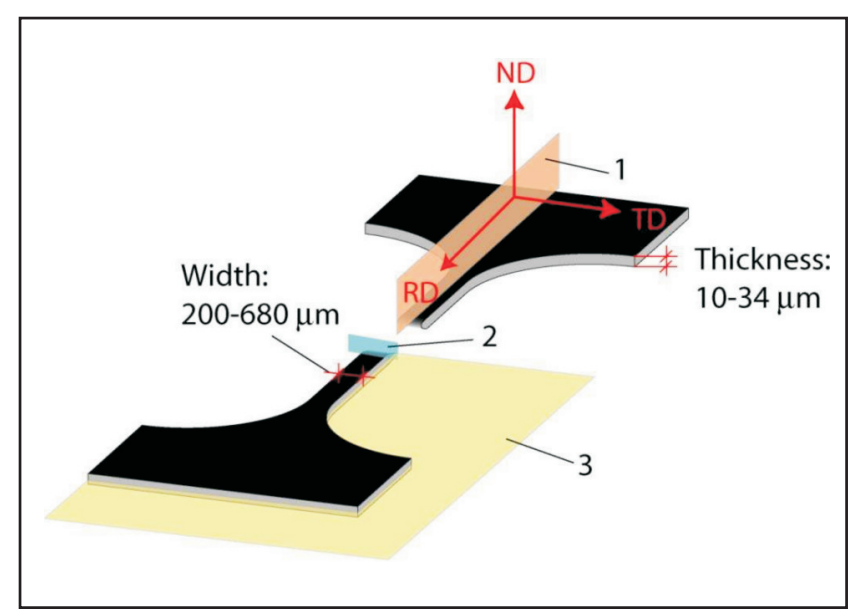

Fig. 1. Sample with the positions of longitudinal (1) and transverse (2) ion beam cutting through a thin copper foil as well as a chemically etched area (3). TD transverse, ND normal and RD rolling direction

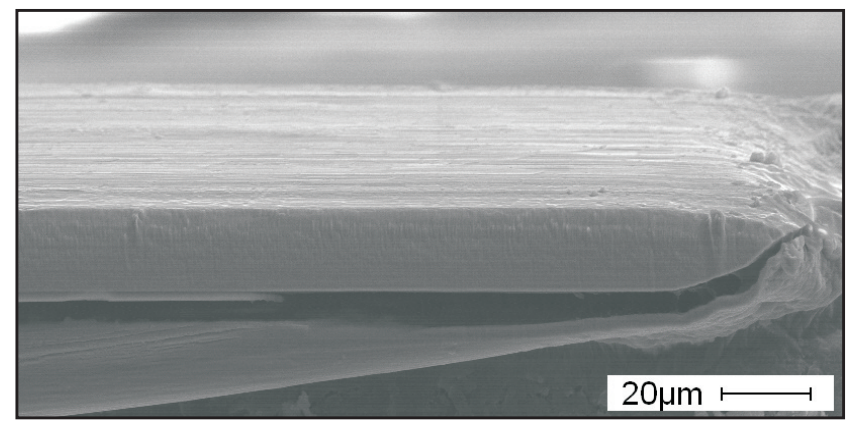

Fig. 3. SEM image of the longitudinal ion beam cut with crack tip

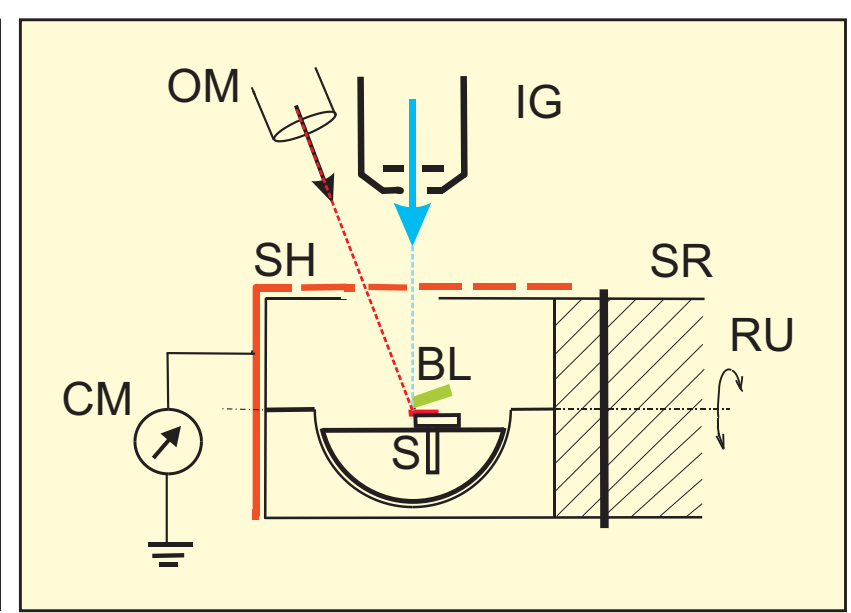

Fig. 2. Gatan PECS with stage for ion beam cutting and the arrangement of ion gun and control attachments: IG ion gun; S sample with support; BL blind; SR sample rod; RU rocking/rotation unit; $\mathrm{SH}$ shutter; OM optical microscope; $\mathrm{CM}$ shutter ion beam current measurement

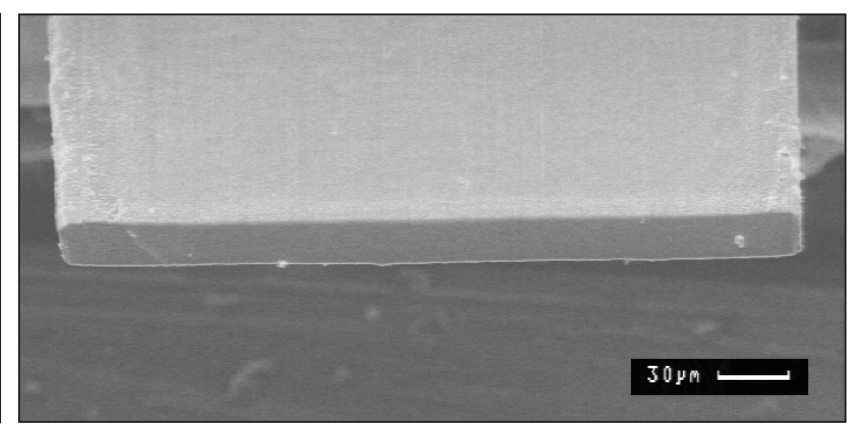

Fig. 4. SEM image of the transverse ion beam cut through a $20 \mu \mathrm{m}$ thick copper foil

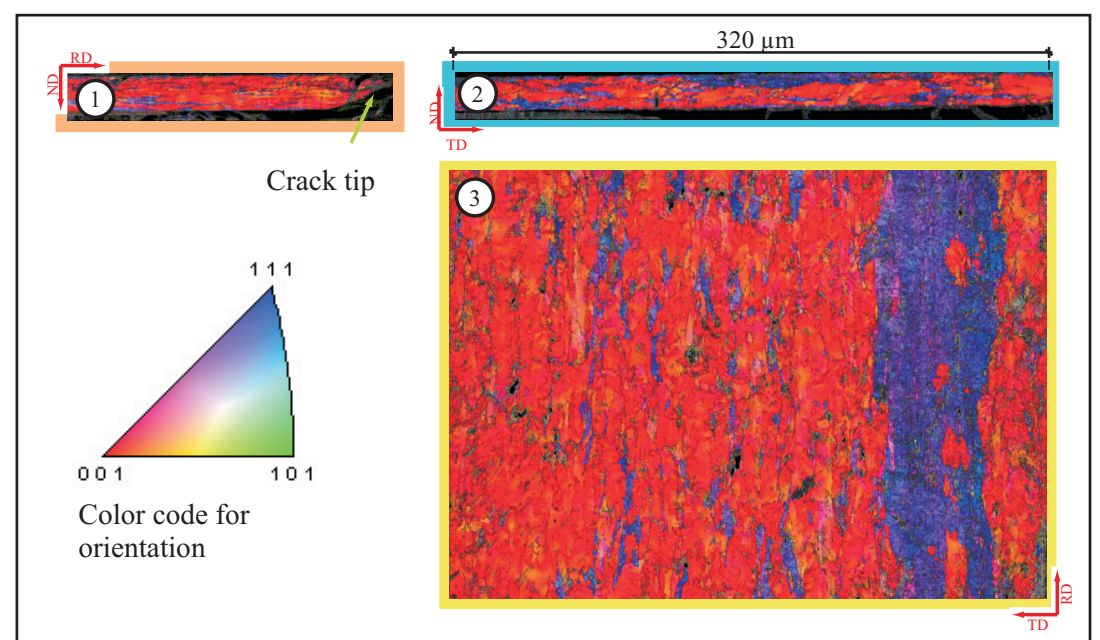

Fig. 5. Crystal orientation maps of three perpendicular sections corresponding to Fig. 1 measured by EBSD

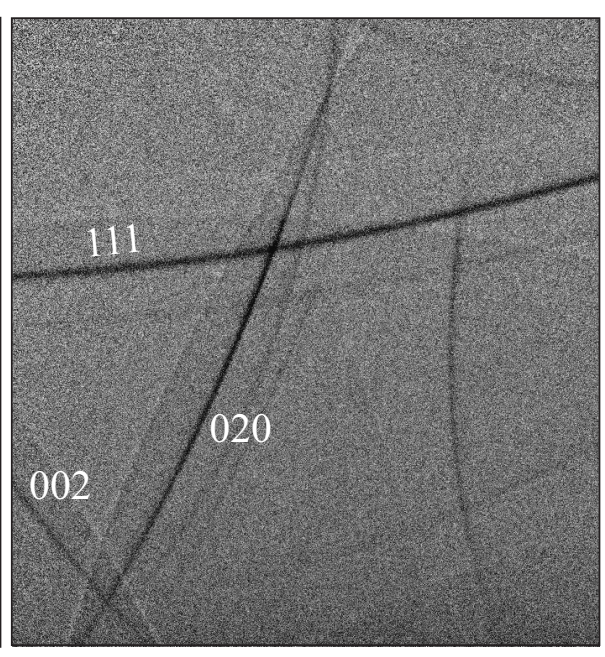

Fig. 6. X-ray Kossel microdiffraction at a deformed Ni crystal 